LINEAR AND NON-LINEAR THEORY OF

GENERALIZED FUNCTIONS AND ITS APPLICATIONS

BANACH CENTER PUBLICATIONS, VOLUME 88

INSTITUTE OF MATHEMATICS

POLISH ACADEMY OF SCIENCES

WARSZAWA 2010

\title{
TENSOR VALUED COLOMBEAU FUNCTIONS ON MANIFOLDS
}

\author{
M. GROSSER \\ Faculty of Mathematics, University of Vienna \\ Nordbergstraße 15, A-1090 Wien, Austria \\ E-mail: michael.grosser@univie.ac.at
}

\begin{abstract}
Extending the construction of the algebra $\hat{\mathcal{G}}(M)$ of scalar valued Colombeau functions on a smooth manifold $M$ (cf. [4]), we present a suitable basic space for eventually obtaining tensor valued generalized functions on $M$, via the usual quotient construction. This basic space canonically contains the tensor valued distributions and permits a natural extension of the classical Lie derivative. Its members are smooth functions depending - via a third slot - on so-called transport operators, in addition to slots one (smooth $n$-forms on $M$ ) and two (points of $M$ ) from the scalar case.
\end{abstract}

In the following, we generalize the construction of the full Colombeau algebra $\hat{\mathcal{G}}(M)$ (see [4]) to the tensor valued case. Let $M$ denote an (orientable) smooth paracompact Hausdorff manifold of dimension $n$; always let $p \in M, f \in \mathcal{C}^{\infty}(M), u \in \mathcal{D}^{\prime}(M), \omega \in$ $\hat{\mathcal{A}}_{0}(M)\left(\subseteq \Omega_{c}^{n}(M)\right) ; R \in \hat{\mathcal{E}}(M)$ (notation as in [4]). Recall the scalar case setting:

Smooth functions : $f \in \mathcal{C}^{\infty}(M)$

Distributions : $u \in \mathcal{D}^{\prime}(M)$

$$
f(p)
$$$$
u(\omega)=\langle u, \omega\rangle
$$

SLOT 1

SLOT 2

Colombeau

generalized functions :

$$
\begin{array}{r}
R \in \hat{\mathcal{E}}(M) \\
R(\omega, p)
\end{array}
$$

SLOTS 1,2

2000 Mathematics Subject Classification: Primary 46F30; Secondary 46T30, 53A45.

Key words and phrases: Colombeau functions, tensors, manifolds.

The paper is in final form and no version of it will be published elsewhere. 
Embedding smooth functions $f$ by $\sigma$ resp. distributions $u$ by $\iota$ into $\hat{\mathcal{E}}(M)$ is effected by using slot 1 resp. slot 2, by means of the formulas (well-known from [2] resp. [4])

$$
\begin{aligned}
(\sigma f)(\omega, p) & :=f(p) \\
(\iota u)(\omega, p) & :=u(\omega)=\langle u, \omega\rangle .
\end{aligned}
$$

Starting from $\hat{\mathcal{E}}(M)$, the Colombeau algebra $\hat{\mathcal{G}}(M)$ is then constructed by passing to quotients of moderate by negligible elements, as usual in Colombeau theory. On the level of quotients resp. classes, $\sigma$ and $\iota$ become equal on $\mathcal{C}^{\infty}(M)$. However, we do not actually perform this last step of the construction at the moment, the question of appropriate basic spaces being our main focus.

For a long period the guiding intuitive idea of the authors of [2] towards obtaining a suitable basic space for tensor valued generalized functions on $M$ had been the following:

for scalars on $M$ use $\hat{\mathcal{E}}(M)$, the candidate which had proven successful in [2];

for tensors on $M$ perform an appropriate "afterward" tensorial construction based on the ready-made space $\hat{\mathcal{E}}(M)$.

All efforts along these lines essentially led to some version of "coordinate-wise embedding" $\iota_{s}^{r}$ of distributional tensor fields of type $(r, s)$ ( $r$ contravariant, $s$ covariant indices). This way of proceeding, however, is ultimately barred due to a consequence of the famous Schwartz type impossibility result: Viewing $\iota$ as a map embedding $\mathcal{D}^{\prime}(M)$ into $\hat{\mathcal{G}}(M)$ as in [2], we have, in general,

$$
\iota(f u) \neq \iota(f) \cdot \iota(u) \quad\left(f \in \mathcal{C}^{\infty}(M), u \in \mathcal{D}^{\prime}(M)\right),
$$

that is, $\iota$ is not $\mathcal{C}^{\infty}(M)$-linear.

To get an impression of what a tensorial construction as just indicated should look like and in which way the above Schwartz type result poses an unsurmountable obstacle to the approach of coordinate-wise embedding we review the situation for tensorial distributions (of type $(r, s)$, say) on $M$. To this end, denote by $\mathrm{T}_{s}^{r} M$ the bundle of $(r, s)$-tensors over $M$ and by $\mathcal{T}_{s}^{r}(M)$ the linear space of smooth sections of $\mathrm{T}_{s}^{r} M$, i.e. of smooth tensor fields of type $(r, s)$ on $M$. The linear space $\mathcal{D}^{\prime r}{ }_{s}(M)$ of tensorial distributions of type $(r, s)$ on $M$ can be defined in several equivalent ways; for our present purpose, we prefer

$$
\mathcal{D}_{s}^{\prime r}(M):=\left(\mathcal{T}_{r}^{s}(M) \otimes_{\mathcal{C}^{\infty}(M)} \Omega_{c}^{n}(M)\right)^{\prime}
$$

(compare section 3.1 .3 of [1] where - due to not assuming orientability of $M$-densities on $M$ take the place of $n$-forms, yielding a slightly more general setting).

Now it is a fundamental result that tensorial distributions can be viewed as tensor fields with (scalar) distributional coefficients ([1], 3.1.15), i.e.,

$$
\mathcal{D}^{\prime r}{ }_{s}(M) \cong \mathcal{D}^{\prime}(M) \otimes_{\mathcal{C}^{\infty}(M)} \mathcal{T}_{s}^{r}(M) \text {. }
$$

A formula completely analogous to (4) is valid (though trivial) on the level of smooth objects:

$$
\mathcal{T}_{s}^{r}(M) \cong \mathcal{C}^{\infty}(M) \otimes_{\mathcal{C}^{\infty}(M)} \mathcal{T}_{s}^{r}(M)
$$

(4) and (5) are interlaced by natural isomorphisms: Denoting the embedding of smooth 
regular objects into distributional ones as

$$
\begin{gathered}
\rho: \mathcal{C}^{\infty}(M) \rightarrow \mathcal{D}^{\prime}(M) \\
\rho_{s}^{r}: \mathcal{T}_{s}^{r}(M) \rightarrow \mathcal{D}^{\prime r}{ }_{s}(M)
\end{gathered}
$$

we obtain the following commutative "TD-diagram":

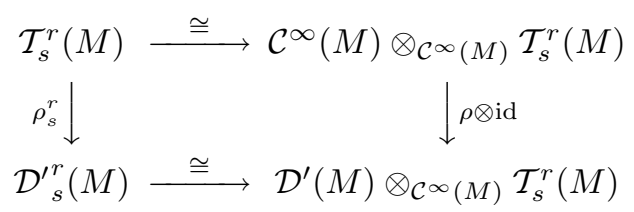

This certainly encourages us to try the definition

$$
\hat{\mathcal{G}}_{s}^{r}(M):=\hat{\mathcal{G}}(M) \otimes_{\mathcal{C} \infty(M)} \mathcal{T}_{r}^{s}(M)
$$

yielding the reassuring "TG-diagram"

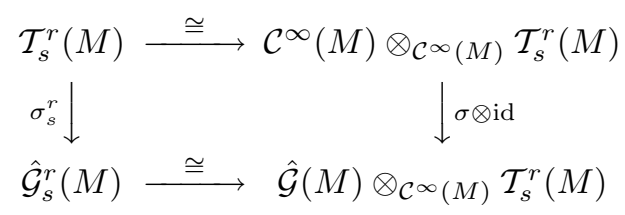

Combining the TD- and the TG-diagrams into one (and omitting the $\mathcal{C}^{\infty}(M)$-subscript at the $\otimes$ sign, as well as all occurrences of " $(M)$ ") results in

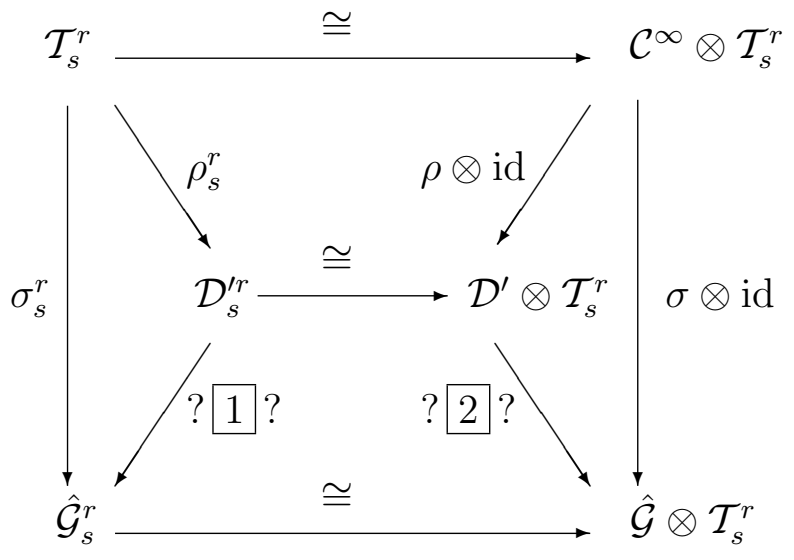

where the arrows denoted by 1 resp. 2 still are waiting to be defined - the former providing the desired embedding of tensor distributions into generalized tensors. Now, 1 certainly would have to be induced by 2 , and for the latter, due to $\sigma=\iota \circ \rho$, the only sensible choice is $\iota \otimes \mathrm{id}$. However, we have to remember that our $\otimes$ signs actually read $\otimes_{\mathcal{C} \infty(M)}$. Therefore, we have to check carefully whether mappings giving rise to a 
commutative "DG-diagram"

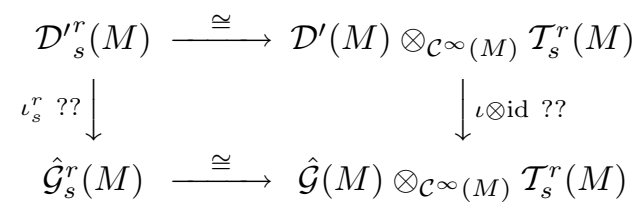

actually exist. Unfortunately, the answer is no! To be sure, on the level of vector space tensor products,

$$
\iota \otimes \mathrm{id}: \mathcal{D}^{\prime}(M) \otimes \mathcal{T}_{s}^{r}(M) \rightarrow \hat{\mathcal{G}}(M) \otimes \mathcal{T}_{s}^{r}(M)
$$

is well-defined. Yet it does not induce a corresponding map on the level of $\mathcal{C}^{\infty}(M)$-module tensor products (which would be what we actually need) since it is not balanced into $\hat{\mathcal{G}}(M) \otimes_{\mathcal{C}^{\infty}(M)} \mathcal{T}_{s}^{r}(M)$ by the Schwartz type theorem:

$$
(\iota \otimes \mathrm{id})((f \cdot u) \otimes t)=\iota(f \cdot u) \otimes t
$$

is different in general (cf. (3)) from

$$
(\iota \otimes \mathrm{id})(u \otimes(f \cdot t))=\iota(u) \otimes(f \cdot t)=(\iota(u) \cdot f) \otimes t=(\sigma(f) \cdot \iota(u)) \otimes t=(\iota(f) \cdot \iota(u)) \otimes t .
$$

It is instructive to take a look at the coordinate version of the preceding (geometrically phrased) impossibility result. As we will show, the attempt to build upon $\iota \otimes$ id is reflected by trying to embed tensor fields coordinate-wise. Again we will arrive at a contradiction, demonstrating that coordinate-wise embedding has to be abandoned completely when spaces of tensor valued Colombeau functions - allowing for a canonical embedding of distributions - are to be constructed.

For localizing, assume that $M$ can be described by a single chart. Then $\mathcal{T}_{s}^{r}(M)$ has a $\mathcal{C}^{\infty}(M)$-basis consisting of (smooth) tensor fields, say, $e_{1}, \ldots, e_{m} \in \mathcal{T}_{s}^{r}(M)$ with $m=n^{r+s}$. By (6), every $u \in \mathcal{D}_{s}^{\prime r}(M)$ can be written as $u=u^{i} \otimes e_{i}$ (using summation convention) with $u^{i} \in \mathcal{D}^{\prime}(M)$. The geometrical requirement of $\iota \otimes$ id being well-defined on the level of module tensor products corresponds to $(\iota \otimes \mathrm{id})(u)$ being independent of basis representation of $u$. Thus consider a change of basis given by $e_{i}=a_{i}^{j} \hat{e}_{j}$, with $a_{i}^{j}$ smooth. Then $u=\hat{u}^{j} \otimes \hat{e}_{j}$ with $\hat{u}^{j}=a_{i}^{j} u^{i}$. Applying $\iota \otimes$ id to either representation of $u$, we obtain

$$
(\iota \otimes \mathrm{id})\left(u^{i} \otimes e_{i}\right)=\iota\left(u^{i}\right) \otimes\left(a_{i}^{j} \hat{e}_{j}\right)=\left(\iota\left(u^{i}\right) a_{i}^{j}\right) \otimes \hat{e}_{j}=\left(\sigma\left(a_{i}^{j}\right) \iota\left(u^{i}\right)\right) \otimes \hat{e}_{j}=\left(\iota\left(a_{i}^{j}\right) \iota\left(u^{i}\right)\right) \otimes \hat{e}_{j}
$$

resp.

$$
(\iota \otimes \mathrm{id})\left(\hat{u}^{j} \otimes \hat{e}_{j}\right)=\iota\left(a_{i}^{j} u^{i}\right) \otimes \hat{e}_{j}
$$

which are different in general due to $\iota\left(a_{i}^{j}\right) \iota\left(u^{i}\right) \neq \iota\left(a_{i}^{j} u^{i}\right)$ (cf. [3 $)$. It should be clear now that relying on coordinate-wise embedding is betting on the wrong horse.

To circumvent this Schwartz type obstacle, the following alternative approach (due mainly to J. A. Vickers and J. P. Wilson, cf. [5]) turned out to be successful eventually: Introduce, in addition to slots 1 and 2 , some slot 3 "inside" of $R$, i.e. intervene "before" $R$ actually acts by assigning some tensor to its argument(s).

From now on, let us write " $t$ " (for "tensor") rather than " $R$ ". Thus the new idea directs us to replace $R(\omega, p)$ by

$$
t(\omega, p, A)
$$


( $A$ having been fed into slot 3 ) in a way that $t$ becomes a member of some space $\hat{\mathcal{E}}_{s}^{r}(M)$ of (smooth) tensor valued functions, to be defined appropriately. This latter space then will serve as the basic space for tensors of type $(r, s)$, consisting of functions having three slots as above.

Observe that this strategy includes "redefining" also the scalar case, in a way that the "old" 2-slot version from [2] resp. [4] has to be upgraded to the "new" 3-slot version. So, strictly speaking the algebra $\hat{\mathcal{G}}(M)$ of (scalar) valued generalized functions discussed in [2] resp. [4] in fact differs (by the absence/presence of slot 3) from the algebra $\hat{\mathcal{G}}_{0}^{0}(M)$ introduced (as the special case $r=s=0$ of $\hat{\mathcal{G}}_{s}^{r}(M)$ ) at the end of this article.

Now let us explain and motivate which kind of objects we should expect to feed into slot 3. As to $\omega$ and $p$, we take $\omega \in \hat{\mathcal{A}}_{0}(M)$ resp. $p \in M$, as we did previously for $R \in \hat{\mathcal{E}}(M)$. $A$, on the other hand, has to be taken as a member of $\Gamma_{c}(\mathrm{TO}(M, M))$, the latter denoting the space of compactly supported smooth sections of the bundle $\mathrm{TO}(M, M)$ of "transport operators" over $M \times M$. More explicitly, $A$ is a compactly supported smooth map

$$
A: M \times M \rightarrow \bigsqcup_{(p, q) \in M \times M} \mathrm{~L}\left(\mathrm{~T}_{p} M, \mathrm{~T}_{q} M\right)
$$

where $\mathrm{L}\left(\mathrm{T}_{p} M, \mathrm{~T}_{q} M\right)$ denotes the space of all linear maps from the tangent space at $p$ to $M$ into the tangent space at $q$ of $M$, and the disjoint union above carries the bundle structure suggested by the obvious local coordinate respresentations. Thus we have, for $p, q \in M$,

$$
A(p, q): \mathrm{T}_{p} M \rightarrow \mathrm{T}_{q} M \quad \text { (linear) }
$$

where $A(p, q)$ smoothly depends on $p$ and $q$.

The new basic space $\hat{\mathcal{E}}_{s}^{r}(M)$ will be defined as a certain subspace (to be specified later) of

$$
\mathcal{C}^{\infty}\left(\hat{\mathcal{A}}_{0}(M) \times M \times \Gamma_{c}(\mathrm{TO}(M, M)), \mathrm{T}_{s}^{r} M\right),
$$

or, with $\hat{\mathcal{B}}(M):=\Gamma_{c}(\mathrm{TO}(M, M))$, of

$$
\mathcal{C}^{\infty}\left(\hat{\mathcal{A}}_{0}(M) \times M \times \hat{\mathcal{B}}(M), \mathrm{T}_{s}^{r} M\right) .
$$

So there remains the question: Why do we introduce slot 3 and how do transport operators enter the scene? The answer is twofold:

- Because it works (in German, we say "Der Zweck heiligt die Mittel", i.e. "The end justifies [sanctifies, literally] the means" in situations like this), i.e. the resulting space $\hat{\mathcal{E}}_{s}^{r}(M)$ permits sensible definitions of

- induced actions of diffeomorphisms $\mu: M \rightarrow N$,

- natural extensions of Lie derivatives $\mathrm{L}_{X}$,

- moderate and negligible elements and, finally

- a space of generalized tensor fields $\hat{\mathcal{G}}_{s}^{r}(M)$ having all the desired properties.

- The introduction of the $A$-slot for tensors is highly plausible - which the remaining part of this article is devoted to convince the reader of.

Let us begin by reviewing the scalar case of embedding a (regular) distribution given by a continuous function $g$ on $M$ into the basic space $\hat{\mathcal{E}}(M)$, using formula 2 for the 
embedding $\iota$ : Pick $g \in \mathcal{C}(M) \subseteq \mathcal{D}^{\prime}(M)$ and think of some $n$-form $\omega$ which approximates the Dirac measure $\delta_{p}$ around $p \in M$; in sloppy notation, $\omega(q) \approx \delta_{p}(q)$ for $p, q \in M$. Then

$$
(\iota g)(\omega, p)=\int_{M} g(q) \omega(q)
$$

collects values of $g$ around $p$ and forms a smooth average (note that $\int \omega=1 !$ ) as value for $(\iota g)(\omega, p)$. Here, $q \mapsto g(q)$ is a scalar valued function on $M$.

Now, if $g$ takes tensors of type $(r, s)$ as values, i.e., if $g$ is a continuous section of $\mathrm{T}_{s}^{r} M$,

$$
q \mapsto g(q) \in\left(\mathrm{T}_{s}^{r}\right)_{q} M,
$$

then the $g(q)$ 's do not live in the same linear space for different $q$ !

In order to average them around $p$, we first have to "gather" them in $p$, i.e. to shift each $g(q)$ from $\left(\mathrm{T}_{s}^{r}\right)_{p} M$ to $\left(\mathrm{T}_{s}^{r}\right)_{q} M$. This is accomplished by $A$ in the following way: For

$$
g(q)=w_{1}(q) \otimes \ldots \otimes w_{r}(q) \otimes \beta^{1}(q) \otimes \ldots \otimes \beta^{s}(q) \in\left(\mathrm{T}_{s}^{r}\right)_{q} M
$$

(where $w_{i}(q) \in \mathrm{T}_{q} M, \beta^{j}(q) \in \mathrm{T}_{q}^{*} M$ for $i=1, \ldots, r, j=1, \ldots, s$ ) we set

$$
B_{s}^{r}(q, p)(g(q)):=A(q, p) w_{1}(q) \otimes \ldots \otimes(A(p, q))^{\operatorname{ad}} \beta^{s}(q) \in\left(\mathrm{T}_{s}^{r}\right)_{p} M .
$$

(The notation $A_{s}^{r}(p, q)$ has to be saved for later use.) So we may form

$$
\left(\iota_{s}^{r} g\right)(\omega, p, A):=\int_{M} B_{s}^{r}(q, p)(g(q)) \omega(q) \in\left(\mathrm{T}_{s}^{r}\right)_{p} M .
$$

In what follows, we will again simply write $\iota g$ for $\iota_{s}^{r} g$. Let us check the status of the objects in the above integrand carefully:

- $B_{s}^{r}(q, p)(g(q))$ is an $(r, s)$-tensor at $p$, depending (smoothly) on $q$;

- $\omega(q)$ (which $q$ viewed as variable) is a compactly supported $n$-form on $M$ with unit integral.

So it seems that the integral on the right hand side of $(7)$ is one of a "new" type (of course, only modulo the previous knowledge of the reader), yet it is perfectly well-defined-just write it out in a chart in the obvious way and check compatibility with chart changes.

As one can show, $\iota g$ as defined above depends smoothly on $\omega, p, A$. (In fact, the proof of this statement represents one of the technically most demanding parts of the forthcoming paper [3].) Thus for each fixed pair $(\omega, A)$ we have that

$$
(\iota g)(\omega, A):=[p \mapsto(\iota g)(\omega, p, A)]
$$

defines a smooth tensor field of type $(r, s)$ on $M$, due to $(\iota g)(\omega, p, A) \in\left(\mathrm{T}_{s}^{r}\right)_{p} M$.

This strongly suggests the following choice for $\hat{\mathcal{E}}_{s}^{r}(M)$ :

$$
\hat{\mathcal{E}}_{s}^{r}(M):=\left\{t \in \mathcal{C}^{\infty}\left(\hat{\mathcal{A}}_{0}(M) \times M \times \hat{\mathcal{B}}(M), \mathrm{T}_{s}^{r} M\right) \mid t(\omega, p, A) \in\left(\mathrm{T}_{s}^{r}\right)_{p} M\right\} .
$$

In particular, $p \mapsto t(\omega, p, A)$ is a member of $\mathcal{T}_{s}^{r}(M)$ for any fixed $\omega, A$. As to the inevitability of requiring smoothness in all three variables for the members of the basic space, see the remarks following formula (2) in [4].

Now, finally, we are going to pass from embedding continuous $g$ 's to embedding distributional tensor fields $u \in \mathcal{D}_{s}^{\prime r}(M)$ into $\hat{\mathcal{E}}_{s}^{r}(M)$.

By definition of $\mathcal{D}^{\prime r}{ }_{s}(M), u$ takes (finite sums of) tensors $\tilde{t} \otimes \omega\left(\tilde{t} \in \mathcal{T}_{r}^{s}(M), \omega \in \Omega_{c}^{n}(M)\right)$ as arguments. 
Now what we need is a good formula for $(\iota u)(\omega, p, A)$. For a definition of $\iota u$ in terms of $u$ we require something that $u$ can properly act upon. We already have $\omega \in \Omega_{c}^{n}(M)$ from slot 1 , so we still to have to make some $\tilde{t} \in \mathcal{T}_{r}^{s}(M)$ enter the scene.

Fortunately, any $t \in \mathcal{T}_{s}^{r}(M)(t=(\iota g)(\omega, A)$ in the case at hand) is completely determined by specifying all contractions $t \cdot \tilde{t} \in \mathcal{C}^{\infty}(M)$ where $\tilde{t}$ runs through $\mathcal{T}_{r}^{s}(M)$. Hence we consider $(\iota g)(\omega, A) \cdot \tilde{t}$ defined pointwise by

$$
\begin{aligned}
((\iota g)(\omega, A) \cdot \tilde{t})(p) & =(\iota g)(\omega, p, A) \cdot \tilde{t}(p) \\
& =\int_{M} B_{s}^{r}(q, p)(g(q)) \cdot \tilde{t}(p) \omega(q) \\
& =\int_{M} g(q) \cdot\left(B_{s}^{r}(q, p)\right)^{\operatorname{ad}}(\tilde{t}(p)) \omega(q) \\
& =\langle\underbrace{g(.)}_{\in \mathcal{D}_{s}^{\prime r}(M)}, \underbrace{\left(B_{s}^{r}(., p)\right)^{\operatorname{ad}}(\tilde{t}(p))}_{\in \mathcal{T}_{r}^{s}(M) \text { for fixed } p} \otimes \underbrace{\omega(.)}_{\in \Omega_{c}^{n}(M)}\rangle
\end{aligned}
$$

(Note that in the third expression of the above calculation, $B_{s}^{r}(q, p)(g(q))$ and $\tilde{t}(p)$ are tensors of types $(r, s)$ and $(s, r)$, respectively, hence their contraction is a scalar resp. a smooth function on $M$. Therefore, the integrals above are usual integrals over $n$-forms rather than of the "new" type discussed above.)

In the last expression above, we are now free to replace the regular distribution $g$ by any $u \in \mathcal{D}_{s}^{\prime r}(M)$. This leads to our definition of $\iota$, finally: Denoting $\left(B_{s}^{r}(q, p)\right)^{\text {ad }}$ : $\left(\mathrm{T}_{r}^{s}\right)_{p} M \rightarrow\left(\mathrm{T}_{r}^{s}\right)_{q} M$ by $A_{s}^{r}(p, q)$, we are led to define

$$
\begin{aligned}
(\iota u)(\omega, p, A) \cdot \tilde{t}(p) & :=((\iota u)(\omega, A) \cdot \tilde{t})(p) \\
& :=\left\langle u, A_{s}^{r}(p, .)(\tilde{t}(p)) \otimes \omega(.)\right\rangle
\end{aligned}
$$

to obtain the desired embedding $\iota=\iota_{s}^{r}: \mathcal{D}_{s}^{\prime r}(M) \rightarrow \hat{\mathcal{E}}_{s}^{r}(M)$.

With this definition of $\iota_{s}^{r}$, the requirement $\hat{\mu} \circ \iota_{s}^{r}=\iota_{s}^{r} \circ \mu^{*}$ (for a diffeomorphism $\mu: M \rightarrow N)$ leads to a sensible definition of $\hat{\mu}: \hat{\mathcal{E}}_{s}^{r}(N) \rightarrow \hat{\mathcal{E}}_{s}^{r}(M)$. The latter, in turn, induces $\hat{\mathrm{L}}_{X}: \hat{\mathcal{E}}_{s}^{r}(M) \rightarrow \hat{\mathcal{E}}_{s}^{r}(M)$ satisfying $\hat{\mathrm{L}}_{X} \circ \iota_{s}^{r}=\iota_{s}^{r} \circ \mathrm{L}_{X}$ and $\hat{\mathrm{L}}_{X} \circ \hat{\mu}=\hat{\mu} \circ \mathrm{L}_{\mu_{*} X}$ (compare [4] for the scalar case).

Corresponding to the above form of $\iota_{s}^{r}$ extending formula (2) to the tensor case, we also have the (much simpler) analog of (1) for embedding smooth tensor fields into the new basic space:

$$
\sigma_{s}^{r}(f)(\omega, p, A):=f(p) \quad\left(f \in \mathcal{T}_{s}^{r}(M)\right) .
$$

Preserving the product of smooth functions in the present context amounts to preserving the tensor product of smooth tensor fields on $M$ when passing to generalized functions via the embedding under discussion. This crucial goal of the Colombeau approach again is achieved by an appropriate quotient construction of moderate modulo negligible members of the basic space.

For a detailed account of the preceding introductory presentation, as well as for an elaboration of the following concluding statement, we refer to the forthcoming paper [3].

With the test for moderateness and negligibility from the scalar case ([2]) suitably adapted to cope with slot 3 , we finally arrive at $\left(\hat{\mathcal{E}}_{s}^{r}\right)_{m}(M), \hat{\mathcal{N}}_{s}^{r}(M)$ and

$$
\iota_{s}^{r}: \mathcal{D}_{s}^{\prime r}(M) \hookrightarrow \hat{\mathcal{G}}_{s}^{r}(M):=\left(\hat{\mathcal{E}}_{s}^{r}(M)\right)_{m} / \hat{\mathcal{N}}_{s}^{r}(M),
$$


together with appropriate actions of diffeomorphisms and Lie derivaties on $\hat{\mathcal{G}}_{s}^{r}(M)$ which naturally extend the corresponding notions on $\mathcal{D}_{s}^{\prime r}(M)$.

Acknowledgments. The author wishes to express his gratitude to the organizers of the GF 07 conference in Bȩdlewo, in particular to Swietłana Minczewa-Kamińska and to Andrzej Kamiński.

This work was supported by projects P-16742 and Y-237 of the Austrian Science Fund.

\section{References}

[1] M. Grosser, M. Kunzinger, M. Oberguggenberger and R. Steinbauer, Geometric Theory of Generalized Functions, Mathematics and its Applications 537, Kluwer Academic Publishers, Dordrecht, 2001.

[2] M. Grosser, M. Kunzinger, R. Steinbauer and J. A. Vickers, A global theory of algebras of generalized functions, Adv. Math. 166 (2002), 179-206.

[3] M. Grosser, M. Kunzinger, R. Steinbauer and J. A. Vickers, A global theory of algebras of generalized functions II: tensor distributions, submitted. Available electronically at http://arxiv.org/abs/0902.1865v1

[4] R. Steinbauer, A geometric approach to full Colombeau algebras, this volume, 267-272.

[5] J. A. Vickers and J. P. Wilson, A nonlinear theory of tensor distributions, ESI-Preprint (available electronically at http://www.esi.ac.at./ESI-Preprints.html) 566, 1998. 